\title{
Creative Design Of Sitting Hug Machine In The Treatment Of Students With Autism
}

\author{
Jih-Sheng Lo ${ }^{1,2}$, Shyh-Chour Huang ${ }^{1}$ \\ ${ }^{1 .}$ Department of Mechanical Engineering, National Kaohsiung University of Applied Sciences, Kaohsiung 80778, Taiwan R.O.C. \\ 2. Department of Digital Content and Technology, National Taichung University of Education, Taichung 40359, Taiwan R.O.C.
}

\begin{abstract}
In recent years, the increasing number of autistic patients has attracted the attention of governments around the world. Normerally, experts will use counseling strategies, drug treatment methods, or the Squeeze Machine, which designed on the basis of the sensory integration theory, to improve those autistic patients' stereotypical behaviors performance. However, we found the existing machine on sale in the market is too bulky, more expensive and not user-friendly for those autistic students to use. Therefore, we had used expert interviews, practical operations, product analysis and then had performed several design improvements experiments, and had successfully completed the innovative prototype named 'Sitting Hug Machine' in an earlier research, and this design has won Taiwan's invention patent certificate recently. The purpose of this paper is to introduce this new design and verify its enhancement and therapeutic effect. We adapted the model of A-B-A-B reversal of Single-Subject Experimental Design(SSED) with two junior high-school autistic students, and we got a lot of good results, including improvement of stereotypical behaviors performance, more flexible operation, lower machine cost and miniaturization of machine volume, etc. Finally, we hope that the results of this study can be further promoted to benefit more autistic patients and improve their stereotypical behaviors.
\end{abstract}

\section{Introduction}

According to official government statistics, the patients with autism in Taiwan had reached 13,684 by 2017 [1]. In an autism survey showed more than $80 \%$ of respondents lacked independent family living skills, that is, they needed more intensive household caretaking [2]. For this reason, we hope the research can provide an effective treatment to help autistic students improve their living quality and learning outcomes and also develope more capabilities. Hence, their family can also efficiently ease the burden of caretaking. Ayres was a pioneer in the treatment of autistic children and the first to propose a theory of sensory integration. Then she further developed an intervention that provides autistic students with a deep pressure on their skin to give them a calming effect [3-6]. Grandin (1992) developed a Squeeze Machine, trying to put the above theory in practice, with its remedy effects described by Ayres validated through a series of experimentations [7-8]. We examined the Squeeze Machine manufactured by Therafin Corporation (as shown in Fig. 1) that was based on Grandin's concept and found that the machine itself has some designs which are not good enough and the some operations are also not patient-friendly. This paper is to introduce this prototype, which we had finished and was named as Sitting Hug Machine as shown in Fig. 2 [9] in an earlier research, and then to verify the therapeutic effectiveness for autistic
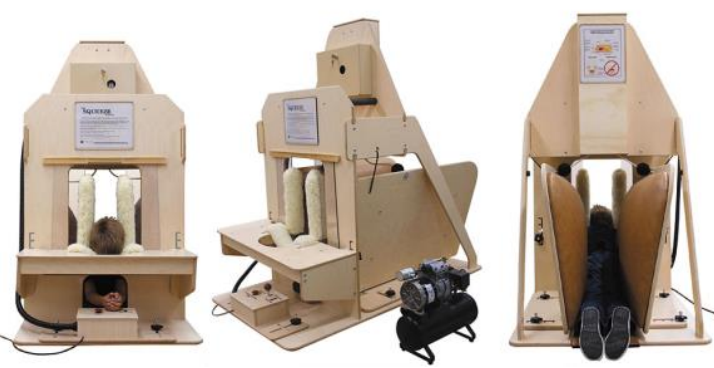

Figure 1. The Squeeze Machine of Therafin Corporation [10]
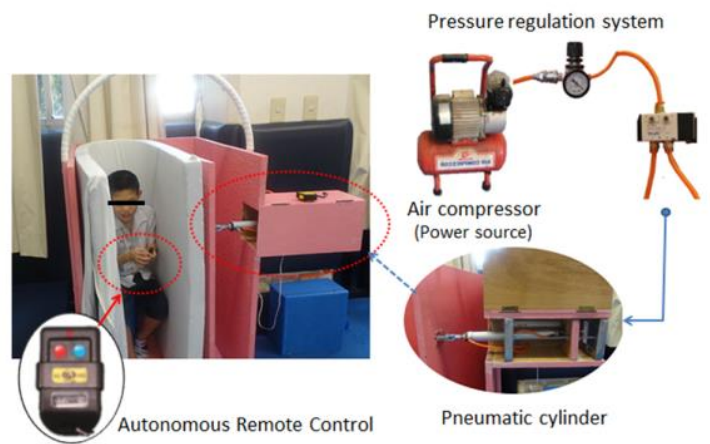

Figure 2. Prototype of the Sitting Hug Machin student regarding this design. 


\section{Developing The Sitting Hug Machine}

In an earlier study of this research, we first examined the Squeeze Machine of Therafin Corporation, and get a conclusion that it is too bulky, expensive and not patientfriendly, etc. Then a series of design and development work were carried out and then an improved design was called 'Sitting Hug Machine' [9]. Ayres (1979) published a theory of Sensory Integration Therapy, and through a series of experimental verification proved it is more effective than the traditional way: through counseling strategies and drug treatment etc. [8]. Then on the basis of this theory, Grandin (1992) designed the first Squeeze Machine, which was the mass produced by Therafin Corporation. This research has selected this machine as a reference model for the innovative design from the beginning [9]. Therefore, the theoretical basis of the Sitting Hug Machine design is based on the Sensory Integration Theory [6]. As Kanner (1943) found, autistic people have three common characteristics: (1) overattachment to an object and failure in social interaction;

(2) late development or inability in language using; and

(3) limited range of repetitive and stereotyped behaviors [11]. Thus American Psychiatric Association (2000) defined these three characters as the core diagnostic features of autism [12]. Stereotypes are the only behavioral characteristics of the three diagnostic features of autism and are therefore the most curable in childhood of autistic patients [13]. Therapists adopt strategies that can effectively improve the stereotypical behavior of students with autism [14]. This research will use the extent of stereotypical behavior as measure indicators of the effectiveness of treatment for autism. Anna Jean Ayres (1968) first found that "learning disabilities" or "Dysfunction in Sensory Integrative Processes" is the root cause of autism, which she later named it "Sensory Integrative Dysfunction" [5], and proposed the Sensory Integration Theory [6]. By applying firm and deep pressure on autism patients' skin, it can ease their tactile defensiveness while increasing their discriminative responses significantly [7]. Therefore, Granting (1992) developed an Squeeze Machine as a remediation tool that provides a deep-pressured hug for autistic students during therapies.

\section{Design Sitting Hug Machine}

Earlier Studies in this Study, We first examined Therafin's Squeeze Machine and conducted in-depth interviews with therapists and patients of the machine. Our conclusion is that it has the following shortcomings: (1). The operating posture is not comfortable, which the patient must squat or tummy, and Children who are too small will not be able to apply; (2). The machine is too bulky and heavy, which needs to be placed in a large space and cannot easily move; (3). Equipment is imported Goods, and the price is very expensive;(4). Non-autonomous control methods, which is a action control box, design in the forefront of the machine outside; and (5). Uneasily adjust the squeeze point with the responding part of body. Thus, an innovative design of the 'Sitting Hug Machine' was finished as an improvement to the above shortcomings of this 'Squeeze Machine' [9]. The prototype machine (as shown in Figure 3 ) uses a thickness of $24 \mathrm{~mm}$ poly-wood frame (which can also be made of lightweight metal truss frame).

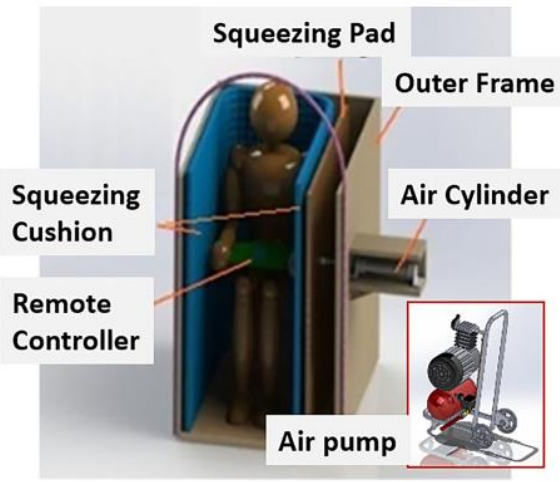

Figure 3. CAD modelling of the Sitting Hug Machine and air pump

The front door is framed by a round stainless steel tube to enhance rigidity of the whole structure and has a pneumatic pump powers to push/pull the press plate. In addition, in normal situation, the patient can sit comfortable on the seat and completely control the squeeze intensity and duration through the wireless remote control, so that the patient can feel much better and relieved. After the patient operating, the cylinder (E) pushes the squeezing pad (F-F) to swing around the center axis (C) to apply the pressing pressure on the pressing pad to give the patient a feeling of hug-like deep pressure on the both sides of shoulders or hip joints of buttocks. In addition, the swinging angle of the pressing pad to the patient can be adjusted according to the patient's feeling. By changing the position of the two squeeze regulators (A and $\mathrm{B}$ ), the pressure exerted on the shoulders and buttocks can be adjusted separately. In addition, the patient may also take different postures, such as sitting down, standing, squatting or other gestures, according to the squeezing position, and then adjust squeezing the whole body, half-length or single hands or feet to achieve a deep feeling of comfort. Patients can also control the squeeze by the remote control the length of time or rhythm as shown in Figure 4.
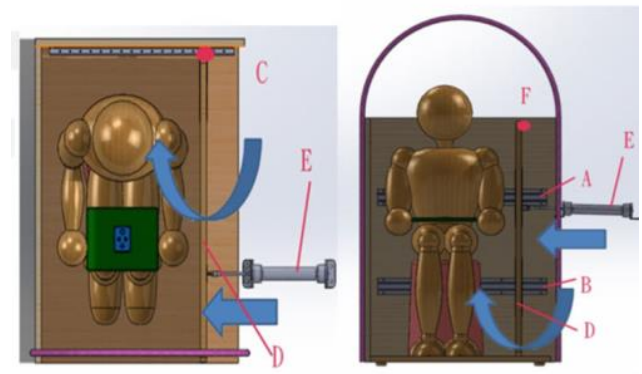

Figure 4. CAD modelling of the Sitting Hug Machine

In summary, the innovation of this innovative design hug machine has the following points: (1).more patient feeling comfortable: Wang (2005) reported her research result, indicating that sitting posture for the patient could be a better design of this machine. This is also the reason 
of the naming as 'Sitting Hug Machine'; (2).more compact size: the original machine design made the patient sit rather than tummy, so the machine was almost half the size of the 'Squeeze Machine'.; (3).Cheaper cost: According to the web page of Therafin Corporation, the price of Squeeze Machine is 4,525 USD, while the cost of our Sitting Hug Machine prototype is about 600 USD approximately;(4). Autonomous control: Autonomous control: While using the Squeeze Machine, the controller is a therapist. If the patient has a hand-held remote controller, and can completely autonomous control the machine, then he or she will feel safer and more comfortable; (5). Easier to adjust: As shown in Figure 3, the squeezing pad (F-F) on the patient's swing angle can be adjusted depending on the patient's feeling. By changing the position of the two squeeze regulators (A and B), the pressure exerted on the shoulders and buttocks of the patient can be adjusted respectively, which gives the patient an extraordinary comfortable feeling. Besides, the patient's posture of squeezing, regardless of sitting down, standing, squatting etc., if it is safe, there will be no limitations

\section{Design validation}

Although the results of our earlier study on the Sitting Hug Machine demonstrated its enhanced effectiveness through the initial in-depth interviews, its validity remains to be further verified in our prototype patient tests. The main purpose of this article is to complete this part of the study:

\subsection{Methodology of experimentations}

In this study, the model of A-B-A-B reversion experimental design model of SSED was adopted to verify the design improvement of the Sitting Hug Machine for Ergonomic and Remedial Ratings. While the Remedial Ratings measured by observing the reduction of stereotypic behavior of the subjects, the Ergonomic Ratings were also measured simultaneously by the questionnaire. Two junior high school students who were rated as having severe and moderate autism respectively enlisted as the subjects in these tests. The test data was further interpreted through the visual analysis and $\mathrm{C}$ statistics to investigate the effectiveness of using the Sitting Hug Machine as the therapeutic tool to reduce stereotypic behaviors of the subjects. The structure of the research method for this experiment was shown in Fig. 4.

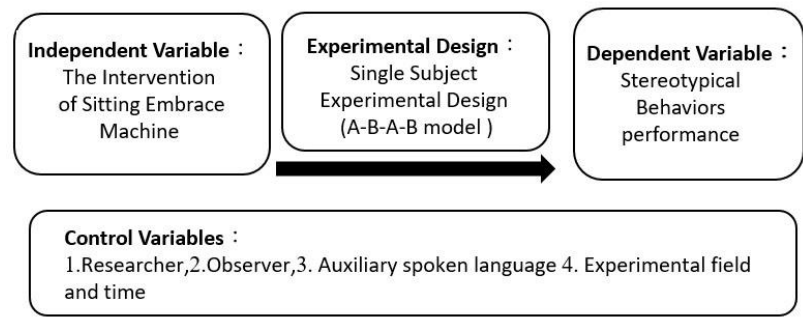

Figure 4. Structure of the research method in this experiment

\subsection{Experimental results}

Due to the limited source of autism cases, it is hard to obtain a large number of experimental samples. Therefore, we took the Purposive Sampling method to investigate the parents of 7 students who had used a Sitting Hug machine and 11 special education teachers who served in the same school. A total of 18 respondents, including 5 males and 13 females, obtained the data shown in Table 1. The test results of the above experiments in terms of Ergonomic and Remedial ratings revealed the facts as follows:

(1). Ergonomic rating: The "Usability" rating of this improved design is 4.10 and 4.03 for its 'Safety', respectively. These results show that the experimental participants were satisfied with the practicality of this machine. However, the score of " accessibility " was 3.92, indicating the need to further strengthen this work.

Table 1. Mean statistical score of operational perception survey.

\begin{tabular}{|c|c|c|c|c|c|}
\hline & \multicolumn{2}{|c|}{ Gender } & \multicolumn{2}{c|}{ Title } & \multirow{2}{*}{$\begin{array}{c}\text { average } \\
\text { value }\end{array}$} \\
\cline { 2 - 5 } & Male & Female & Teacher & Parents & 4.10 \\
\hline Usability & 4.36 & 3.92 & 3.98 & 4.14 & 3.92 \\
\hline accessibility & 4.09 & 3.79 & 3.82 & 3.96 & 4.03 \\
\hline Safety & 4.13 & 3.96 & 3.98 & 4.05 & 4.92 \\
\hline
\end{tabular}

(2). Intervention effectiveness analysis : In this study, two subjects were recruited at each stage (baseline period (A1), first interventional period (B1), reversion stage (A2). The data of the second intervention period (B2) are presented with the correct rate curve (Fig. 5, 6); and the visual analysis method is further adopted. The testers were included in the summary table during each stage and between stages; they were tested with C-statistics to investigate the effect of Sitting Hug Machine intervention on stereotypical behaviors of the two subjects.

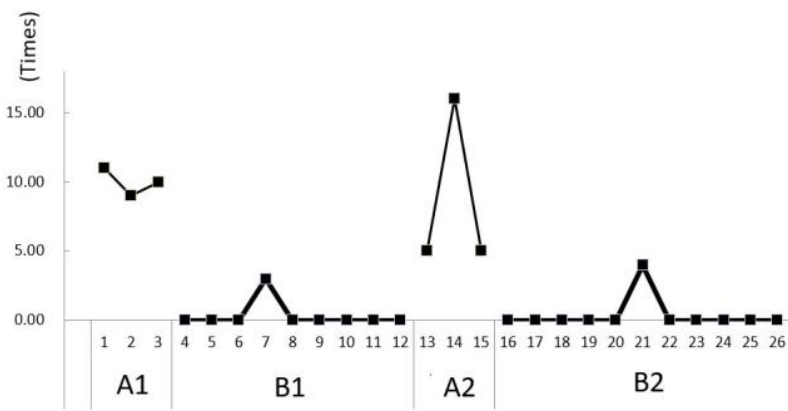

Figure 5 curves of the number of stereotypical behaviors before and after using a Sitting Hug Machine (subject A) 


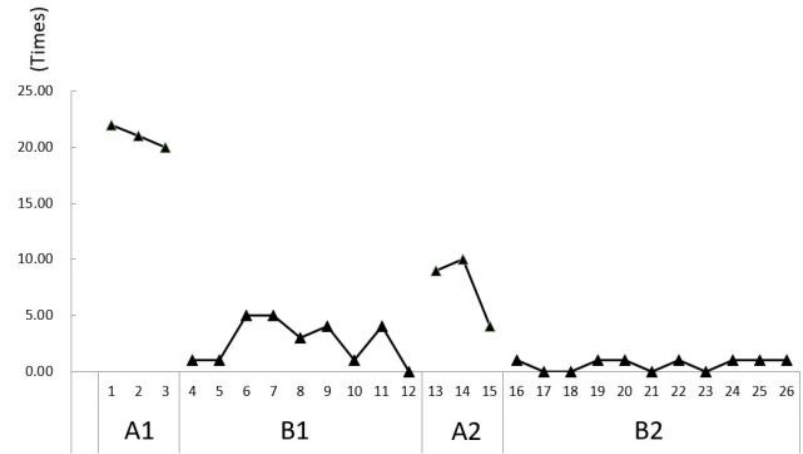

Figure 6 curves of the number of stereotypical behaviors before and after using a Sitting Hug Machine (student B)

Regarding the remedial effect of reducing stereotyped behavior in subjects, subject A reduced by 9.67 times and subject $\mathrm{B}$ by 18.33 times. Therefore, the $\mathrm{z}$-scores of $\mathrm{C}$ statistic in the first stage of experimental design A1 + B1 are 2.73 and 2.78 , respectively, which are both greater than 0.01 , reaching a significant level. This result indicated that the remedial effect of the Sitting Hug Machine was confirmed. Then in the second stage, the reductions in stereotypic behaviors are 7.22 and 7.11 times, and the $\mathrm{z}$-scores of $\mathrm{C}$ statistic in the second stage of reversal and experimental designs $\mathrm{A} 2+\mathrm{B} 2$ are 1.12 and 3.06 respectively, both being more than 0.01 again and thus reached the level of significance. This result further confirms the remedial effect of the Sitting Hug Machine.

\subsection{Discussion}

In summary, which the test results described above, the following points on this research could be confirmed:

(1). Compared with the Squeeze Machine, the improvements of the Sitting Hug Machine regarding the Experimental participants feeling more comfortable, the compact size and the light weight, the cheaper cost, and the autonomous control were all confirmed by therapists and patients. Only the enhancement in easy adjustment requires further advancements. Therefore, the design of Sitting Hug Machine could be assessed as successful.

(2). The remedial effectiveness of the Sitting Hug Machine is validated. The results are in line with the findings of Edelson et al. (1999) that is beneficial to increase the depth of pressure by the calming effect on children with autism.

(3). According to the tutor's observation records, the degree of concentration of subjects was significantly improved. However, the supervisor's observation records will be more subjective. Therefore, in order to further verify this result, more objective data may be needed.

\section{Conclusions}

This paper had successfully validated that the enhanced design of the Sitting Hug Machine developed in our former study is effective. We had made quite a dramatic improvement on this Machine, and also highly affirmed by the students, parents and special education teachers who participated in the experiment. For they found that after the experiment, students' stereotypes behaviors have been greatly improved. We not only had exhibited effectiveness in its improvements on the existing Squeeze Machine, but also had been in its remedial effects on autistic students, and this design has won Taiwan's invention patent certificate in 2015.Therefore, this Sitting Hug Machine is recommended for students with autism, and we might further commercialize it to benefit more patients.

\section{References}

1. Ministry of Health \& Welfare of R.O.C., The Disabled Population. Retrieved April 18, 2017, from http://www.fact.org.tw/index.php/politic-2/2016-1005-08-16-50/303-8787jhhhgg67676 (2017)

2. Chang, C. F., Exploring the Function of Problematic Behaviors of Children with Autism],Bulletin of Special Education], 18, 127-150 (2000)

3. Ayres, A. J., Sensory integrative processes and neuropsychological learning disability. Learning Disorders, 3, 41-58 (1968)

4. Ayres, A. J.,Types of sensory integrative dysfunction among disabled learners. American Journal of Occupational Therapy, 26(1), 13-18 (1972)

5. Ayres, A. J., Sensory integration and the child (1st Ed.). Los Angeles, CA: Western Psychological Services (1979)

6. Ayres, A. J., Sensory integration and the child: understanding hidden sensory challenges (25th Anniversary Ed., Revised and updated by Pediatric Therapy Network). Los Angeles, CA: Western Psychological Services (2005)

7. Grandin, T., Calming Effects of Deep Touch Pressure in Patients with Autistic Disorder, College Students, and Animals. Journal of Child and Adolescent Psychopharmacology, 2(1), 63-72 (1992)

8. Edelson, S. M., Edelson, M. G., Kerr, D. C., \& Grandin, T., Behavioral and physiological effects of deep pressure on children with autism: a pilot study evaluating the efficacy of Grandin's Hug Machine. The American Journal of Occupational Therapy, 53(2), 145-152 (1999)

9. Lo, J. S., Lee, K. C. K., \& Wang J. X., Designing a Sitting Hug Machine for Pupils with Autism. Proceedings from KEER2016: International Conference on Kansei Engineering and Emotion Research, University of Leeds, Leeds, UK: European Kansei Group. (Listed as 048.pdf in: https://drive.google.com/drive/folders/0B714UtIzyA G4U3Ztc1Z0MXNkNE0 ) (2016)

10. Therafin Corporation, Squeeze Machine [Introduction]. Retrieved from http://www.therafin.com/squeezemachine.htm (2017, June 16) 
11. American Psychiatric Association, Diagnostic and statistical manual of mental disorders (DSM-IV-TR). Washington DC: American Psychiatric Association (2000)

12. LaGrow, S. J., \& Repp, A.C., Stereotypic responding: A review of intervention research. American Journal of Mental Deficiency, 88(6), 595-609 (1984)

13. Anderson, C., Behavioral Therapies: Key Interventions in ASD. Interactive Autism Network. Retrieved June 16, 2017, from https://iancommunity.org/cs/simons_simplex_comm unity/behavioral_therapies (2012)

14. Wang, C. L., The Effects of Deep Pressure Touch Treatment of Anxiety of Children with Autism]. Master Thesis, The Department of Physical Therapy and Assistive Technology, National Yang-Ming University, Taiwan] (2005) 